\title{
The CERN-EU high-energy Reference Field (CERF) facility: applications and latest developments
}

\author{
Marco Silari, ${ }^{1, *}$, and Fabio Pozzi ${ }^{1}$ \\ ${ }^{1}$ CERN, 1211 Geneva 23, Switzerland
}

\begin{abstract}
The CERF facility at CERN provides an almost unique high-energy workplace reference radiation field for the calibration and test of radiation protection instrumentation employed at high-energy accelerator facilities and for aircraft and space dosimetry. This paper describes the main features of the facility and supplies a non-exhaustive list of recent (as of 2005) applications for which CERF is used. Upgrade work started in 2015 to provide the scientific and industrial communities with a state-of-the-art reference facility is also discussed.
\end{abstract}

\section{Introduction}

Neutron calibrations often need to be performed with neutron energies or spectral distributions very much different from those generated by radioactive sources such as Am-Be and Cf-252, employed in standard calibration laboratories. This is also highlighted by refs. [1] and [2], which call for the simulation of the neutron energy spectra encountered in the vicinity of high-energy accelerator facilities and in high-flying aircrafts, since high-energy neutrons can deliver a significant fraction of the ambient dose equivalent.

The CERF facility [3], set-up at CERN in the early nineties, provides mixed radiation fields representative of those found outside the shielding of high-energy hadron accelerators and sufficiently similar to the cosmic ray field encountered at $10-20 \mathrm{~km}$ altitude. This allows instrumentation to be tested, inter-compared and calibrated at CERN and subsequently used for either inflight measurements on aircrafts or radiation protection surveys around particle accelerators. CERF has also been employed for a variety of other applications such as radiobiology studies, cross section measurements, investigation of activation of accelerator materials, intercomparison of individual dosimeters, and benchmarking of Monte Carlo codes against experimental data (see Section 3). This work describes the facility, lists its main applications, provides some representative results and illustrates the upgrade program started in 2015 to provide the scientific and industrial communities with an improved, state-of-the-art reference workplace facility.

\section{Description of the facility}

\subsection{Set-up}

Figure 1 shows the detailed geometry of CERF as simulated in the FLUKA Monte Carlo code $[4,5]$. CERF is located in the North Experimental Area on the CERN Prévessin site. The CERF hadron beam is produced by the primary $450 \mathrm{GeV} \mathrm{c}^{-1}$ proton beam from the Super Proton Synchrotron (SPS) on the T4 production target. The secondary beam originating from the T4 target is composed of $61 \%$ positive pions, $35 \%$ protons and $4 \%$ positive kaons [6] with a momentum of $120 \mathrm{GeV} \mathrm{c}^{-1}$. To calculate the beam composition at the CERF irradiation area, one must take into account that $4.2 \%$ and $27 \%$ of the pions and kaons, respectively, decay over the approximately $410 \mathrm{~m}$ long transfer line between T4 and CERF. Hence, the actual beam composition is $60.7 \%$ positive pions, $36.3 \%$ protons and $3.0 \%$ kaons. The hadron beam impinges on a copper target, $7 \mathrm{~cm}$ in diameter and $50 \mathrm{~cm}$ in length, which can be installed under either a concrete roof $80 \mathrm{~cm}$ thick or an iron roof $40 \mathrm{~cm}$ thick. The secondary particles produced in the target (mostly neutrons and photons, with a few percent contribution from electrons, protons, pions and muons) traverse these roof shields producing almost uniform radiation fields over two areas of $2 \times 2 \mathrm{~m}^{2}$ located at approximately $90^{\circ}$ with respect to the incoming beam direction. Each $2 \times 2 \mathrm{~m}^{2}$ area is divided into 16 squares of $50 \times 50 \mathrm{~cm}^{2}$, and each element of this grid represents a reference exposure location. Additional reference positions are available behind the lateral shielding of the irradiation area, at the same angles with respect to the target as for the two roof positions. The nominal measurement locations are at the centre of each square at $25 \mathrm{~cm}$ height above the roof [3] or $25 \mathrm{~cm}$ distance from the lateral shielding, respectively. The copper target can also be removed to allow instrumentation or other targets to be directly placed in the beam, as for cross section measurements (see Section 3.5).

\footnotetext{
* Corresponding author: Marco.Silari@.cern.ch
} 


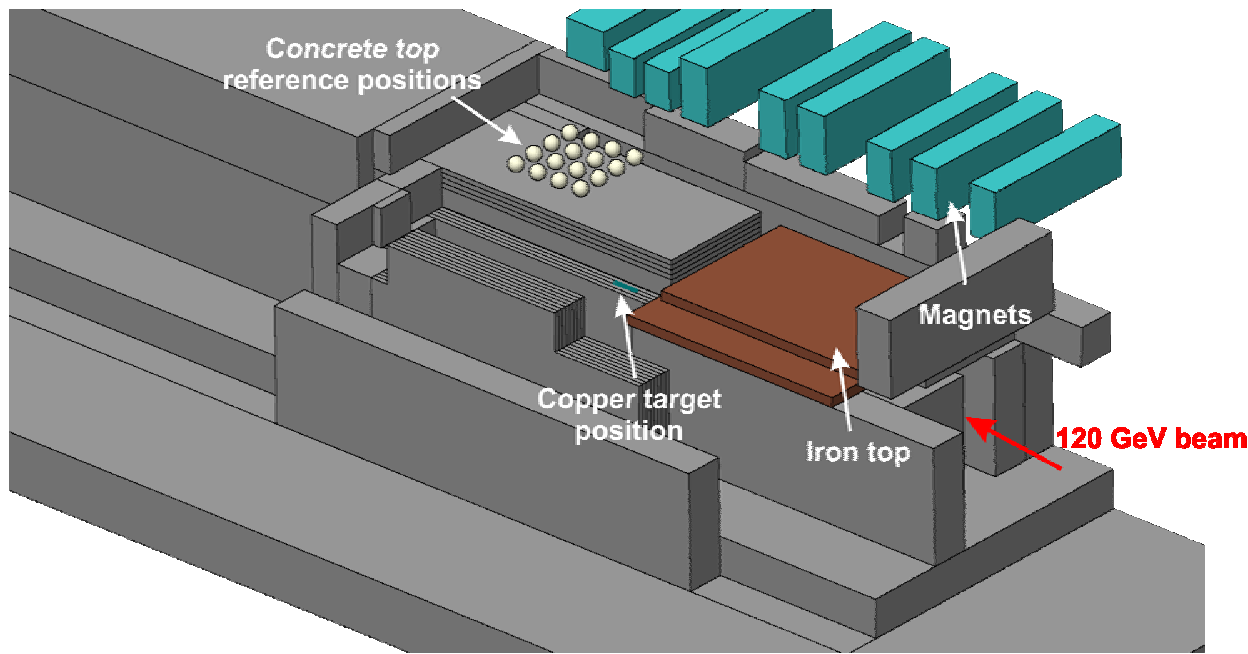

Figure 1. Geometry of CERF as simulated with FLUKA. Here, the copper target is placed under the concrete roof.

\subsection{Beam monitoring and imaging}

The beam is delivered to CERF with a typical intensity in the range $10^{6}$ to $10^{8}$ particles per SPS beam extraction (spill), with two to three beam extractions of about $5 \mathrm{~s}$ duration over an SPS cycle that can vary in the range 30$45 \mathrm{~s}$. During the extraction time the beam intensity is constant resulting in a constant radiation field at the exposure positions. The beam monitoring, on which the normalization of all measurements relies, is provided by an air-filled, parallel-plate, transmission-type ionization chamber (IC); the IC calibration factor was recently verified by means of the multi-foil activation technique using two reactions: the well-known ${ }^{27} \mathrm{Al}\left(\mathrm{p}, 3 \mathrm{pn},{ }^{24} \mathrm{Na}\right.$ monitor reaction and the alternative ${ }^{n a t} \mathrm{Cu}(\mathrm{p}, \mathrm{x})^{24} \mathrm{Na}$ reaction $[7,8]$. These experimental values were compared with the calibration factor obtained simulating with FLUKA the expected charge collected on the plates of the IC; the agreement is very satisfying [7]. One ICcount corresponds to $22000 \pm 2200$ particles impinging on the target.

Typical values of ambient dose equivalent rates are 0.2-0.3 nSv per IC-count over the 16 reference exposure locations on the concrete roof and 1-1.5 nSv per ICcount on the iron roof. By assuming an average of three 5 -second spills per minute and adjusting the beam intensity on the target, one can obtain ambient dose equivalent rates approximately from $5 \mu \mathrm{Sv} \mathrm{h}{ }^{-1}(30 \mathrm{nSv}$ per spill) to $250 \mu \mathrm{Sv} \mathrm{h}^{-1}(1.5 \mu \mathrm{Sv}$ per spill) on the concrete roof and side, and from $18 \mu \mathrm{Sv} \mathrm{h}^{-1}(100 \mathrm{nSv}$ per spill) to $360 \mu \mathrm{Sv} \mathrm{h}^{-1}$ ( $2 \mu \mathrm{Sv}$ per spill) on the iron roof. To provide an independent reference and to monitor the beam stability, the CERN rem counter LINUS $[9,10,11$, 12] is placed in a fixed position on either the concrete or iron roof (according to where measurements are being performed). The steady value of $\mathrm{H}^{*}(10)$ per IC-count measured by the LINUS indicates stable beam conditions. The example in Figure 2 shows how the statistical fluctuations of the LINUS reading correctly decrease with increasing beam intensity while the average remains constant. At about 100 IC-counts per spill the LINUS count rate is approximately 20 counts per spill (for a LINUS sensitivity of 0.92 counts per $n S v$ and an $\mathrm{H}^{*}(10)$ value of about $0.2 \mathrm{nSv}$ per IC-count on concrete top, CT, position 14) and it is 50 times higher at 5000 IC-counts per spill. A change in the LINUS reading would point to a mis-steering of the beam on the target.

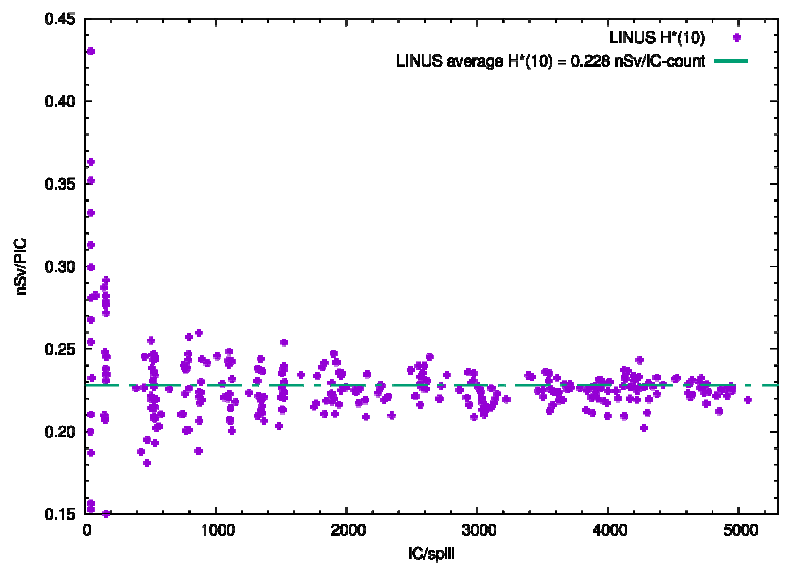

Figure 2. $H^{*}(10)$ rate (normalized to the IC-count rate) measured by the LINUS placed in CT14 as a function of the IC count rate and the average value.

The target alignment is made by exposing Polaroid films for a few minutes to the beam in order to integrate a fluence of about $10^{4} \mathrm{IC}$-counts (Figure 3). The beam is Gaussian and its rectangular shape, mostly contained in an area of about $3.5 \mathrm{~cm}$ wide and $3 \mathrm{~cm}$ high (at the maximum beam intensity) or smaller, is defined by a set of collimators in the T4-CERF beam line. After the target has been aligned, during measurements the beam profile can be routinely checked by a multi wire proportional chamber (MWPC) installed between the two target positions (Figure 4). Recently, a GEM detector was installed to monitor the alignment of the beam with respect to the target (see Section 4.1). 

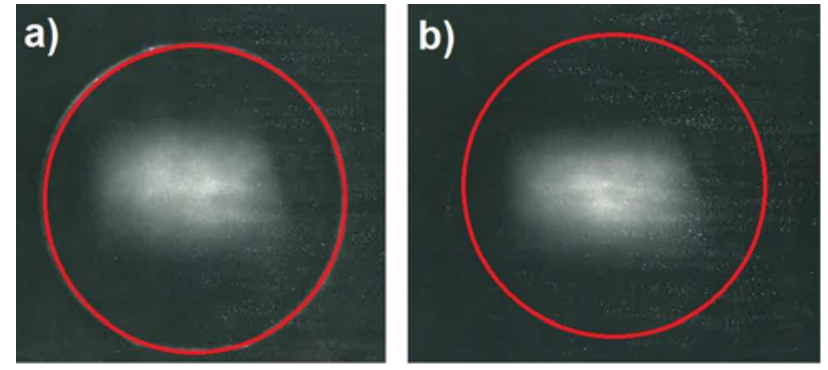

Figure 3. Polaroid views of the beam spot at the front (a) and exit (b) face of the target. The target diameter is indicated by the red circle.

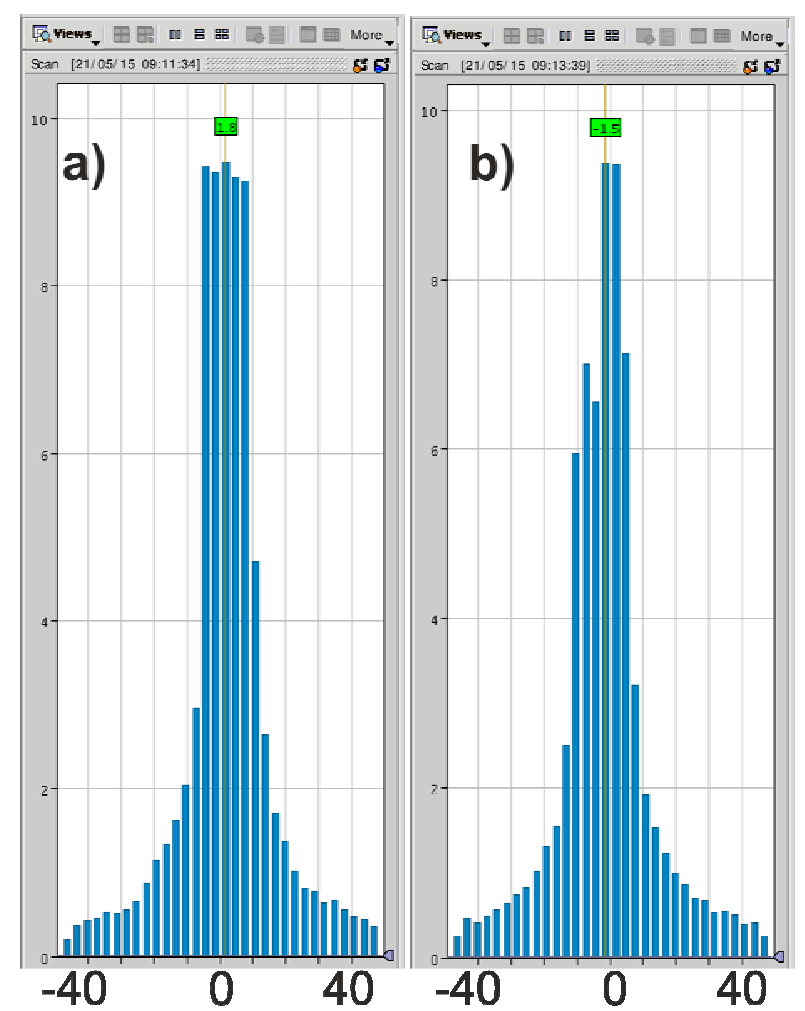

Figure 4. MWPC beam profiles, vertical (a) and horizontal (b). The $\mathrm{x}$-axis scale is in $\mathrm{mm}$.

\subsection{Muon background}

A significant low-LET background radiation field is present on the concrete and iron roof-shields. This background is mainly due to high-energy muons originating from the pion decay in the beam line. It can result in an apparent non-linearity of a detector response when measurements are carried out at different intensities of the incident hadron beam. This effect must be taken into account when measurements are performed by means of ionisation chambers, TEPC and other active devices that have considerable sensitivity to low-LET radiation. No significant muon background is present at the side concrete shielded positions. For more information on the muon background component and how to subtract it, the reader can refer to [3] and the references there-in quoted.

\section{Applications}

To show the range of uses of CERF, this section gives a brief, non-exhaustive overview of the most important and recent applications (as of 2005), together with a few representative results. An overview of earlier experiments can be found in ref. [3].

\subsection{Test, workplace field calibration and intercomparison of radiation protection instrumentation}

In 2007 the performances of conventional and extendedrange rem-counters and of a tissue-equivalent proportional counter (TEPC) were tested for their use around high-energy accelerators [13]. The results confirmed the reliability of the extended-range remcounters and TEPC, which are specifically designed to operate in these conditions. The conventional remcounters, designed for neutron energies up to $15 \mathrm{MeV}$, substantially underestimated the neutron $H^{*}(10)$. However, a field calibration factor for each detector can be deduced at CERF; this should be used when these devices are employed in a similar high-energy field.

A similar experiment was conducted in 2012 by Caresana et al. [14] to investigate the performances of conventional and extended-range rem-counters including a novel instrument called LUPIN, two TEPCs and bubble detectors. The results were also compared with the FLUKA reference values [3]. The conventional remcounters were in good agreement within their uncertainties and underestimated the $\mathrm{H}^{*}(10)$ as measured by the extended-range instruments and as predicted by FLUKA. The TEPCs slightly overestimated the FLUKA value and indicated that the non-neutron part of the stray field accounts for about $30 \%$ of the total $\mathrm{H}^{*}(10)$.

Unlike the two above-mentioned studies, which were carried out on the concrete roof, the following experiments were performed inside the irradiation cave to evaluate the capabilities of potential beam monitors. Vincke et al. [15] experimentally and theoretically studied the response to several types of incident particles of a PMI ionisation chamber, designed for use in the Large Hadron Collider (LHC) to obtain an indication of the machine performance via a measurement of radiation under beam operation conditions. This study also demonstrated that the FLUKA code is well suited to predict the response of ionisation chambers in a mixed radiation field.

Aza et al. [16] investigated the use of a triple GEM detector designed for low-energy neutrons as secondary on-line monitor, measuring the low-energy neutron flux scattered from the target inside the CERF irradiation area.

Ytre-Hauge et al. [17] investigated the capability of detecting neutrons by a monitor based on the registration of radiation effects in Static Random Access Memories (SRAMs). The SRAMs were irradiated in beams lines at PTB, Braunschweig, Germany, at the Oslo Cyclotron Laboratory, Norway, at the Svedberg Laboratory in Uppsala, Sweden, at the Institute for Energy Technology, Kjeller, Norway, and at CERF. 
Haninger et al. [18] tested and calibrated a new albedo neutron dosemeter based on TLD, which was introduced by the individual monitoring service of the Helmholtz Zentrum Munchen. The field calibration revealed that this kind of dosemeter can also be used for personal monitoring at workplaces of high-energy particle accelerators.

\subsection{In-flight and in-space measurements based on CERF calibration}

Since the CERF mixed field is similar to the one encountered at aircraft altitudes, several studies and instrument calibrations were performed over the past 20 years, of which we provide a few examples. Vuković et al. [19] investigated the use of a combination of track etch detectors (CR-39, LR-115) with a plastic converter or boron foil. These detectors were calibrated at CERF as well as in a nuclear reactor before their use on aircraft.

Jadrníčková and Spurný [20] tested a LET spectrometer based on track detectors on the International Space Station (ISS), comparing the results with the measurements performed at CERF. The LET distributions of dose equivalent obtained at CERF and on-board ISS agree very well within their uncertainties, making CERF a suitable calibration facility for in-space dosimetry. The Timepix detector, which is currently employed on the ISS for the measurements of the dose to astronauts [21], was also tested at CERF for its use as a single dosimeter and LET spectrometer in mixed radiation fields created by heavy ions [22].

\subsection{Dosimeter intercomparison}

In 2014 a dosimeter intercomparison was organized at CERF. Seven U.S. laboratories participated sending their dosimeters to CERN (Table 1). Four prior comparisons were performed in the past to evaluate the state of personnel dosimetry implemented at accelerator facilities at the U.S. Department of Energy (USDOE) laboratories. Since the last test in 1999 , the dosimetry technology has changed to some degree and USDOE regulations in the U.S. have adopted dose equivalent quantities specified in the ICRP 60. In addition, many laboratories have moved to the use of vendor-provided dosimetry. The aim of this study was to evaluate the current state of dosimetry related to high-energy neutron personnel monitoring [23].

Table 1. List of the US laboratories participating in the 2014 individual dosimetry intercomparison.

\begin{tabular}{|l|}
\hline Laboratory \\
\hline Brookhaven National Laboratory \\
\hline Thomas Jefferson National Accelerator Facility \\
\hline Pacific Northwest National Laboratory \\
\hline Idaho National Laboratory \\
\hline Los Alamos National Laboratory \\
\hline Nevada National Security Site \\
\hline Landauer, Inc. \\
\hline
\end{tabular}

Recently, the CERF facility has been employed for the measurements of spallation cross sections in thin $\mathrm{Fe}, \mathrm{Ti}$ and $\mathrm{Cu}$ targets $[24,25]$. Thin foils were exposed to the CERF hadron beam and irradiated for about 8 hours to obtain measurable activities. The spallation cross sections for protons and pions at $120 \mathrm{GeV} \mathrm{c}^{-1}$ were derived from $\gamma$-spectrometry measurements and FLUKA simulations; they were compared with the existing values in the literature and the fragmentation and factorization hypothesis were evaluated.

Additional measurements of spallation cross sections on other target materials are planned for the near future. In order to obtain absolute cross sections, the possibility of using a $100 \%$ negative pion beam is currently under investigation; this will avoid having to resort to the Monte Carlo method to obtain the proton and pion cross sections, which are otherwise derived from mixed pion/proton irradiation.

\subsection{Other applications}

Over the years CERF has also been used for a number of other applications:

- Shielding experiments [26], which allowed measuring the inelastic attenuation length of high energy neutrons in a thick iron shield.

- Performance test of beam loss monitors, which are essential tools to protect accelerator components from damage due to unforeseen, critical beam losses [27].

- Measurements of Single Event Upsets (SEUs) for the ESA SEU Monitor [28] and Single Event Effects (SEEs) for a new platform dedicated to the long-term characterization of the atmospheric natural radiative environment at mountain altitude [29].

- Benchmark of Monte Carlo codes against experimental data [30].

\section{Latest upgrades}

Since 2015 an upgrade work has been undertaken, starting with the installation of improved target supports to make the target alignment to the beam easier and more accurate. A new up-to-date website [31] is currently maintained; this provides important information concerning the facility, access and safety rules, beam time and schedule, and reference articles.

\subsection{New beam imaging and monitoring systems}

A new beam imaging system employing a triple-GEM detector was tested in view of substituting the Polaroid films for target alignment [32] (section 2.2). The GEM system is mounted on both the downstream and upstream end of a hollow tube of the same dimensions of the copper target. This device allows a real time imaging of the beam spot on the target (Figure 5) providing a 2D image of the beam (Figure 6). This system thus allows a more accurate and rapid alignment of the beam on the copper target as compared to the films. 


\section{Gem on target Upstream}

\section{Gem on target Downstream}
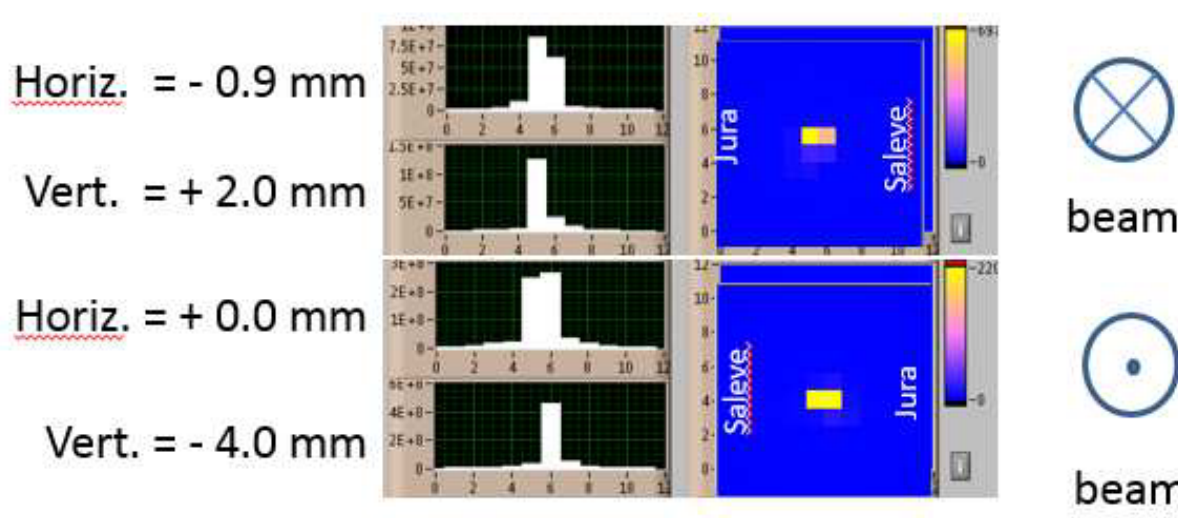

beam

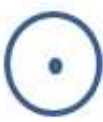

beam
Figure 5. Position of the beam with respect to the target axis as measured by the triple-GEM detector.

In addition, the use of the GEM detector as beam intensity monitor was investigated, showing a satisfactory agreement with the measurements provided by the IC [32]. However, the triple-GEM has not yet shown to provide the same accuracy and stability of the IC as reference monitor to count beam particles. Since the IC is a single point of failure, options for a back-up solution are currently under investigation.

\subsection{New FLUKA reference simulations}

Measurements at CERF rely on the accurate characterization of the radiation field. FLUKA simulations of the entire facility providing reference neutron $H^{*}(10)$ values and spectral fluence at each exposure location were performed in 1992 (quoted in ref. [3]) and have been used since that time. Improvements of the physics models (especially concerning the neutron transport) were included in FLUKA since then, so that some variations in the simulation results can be expected with the latest version of the code.
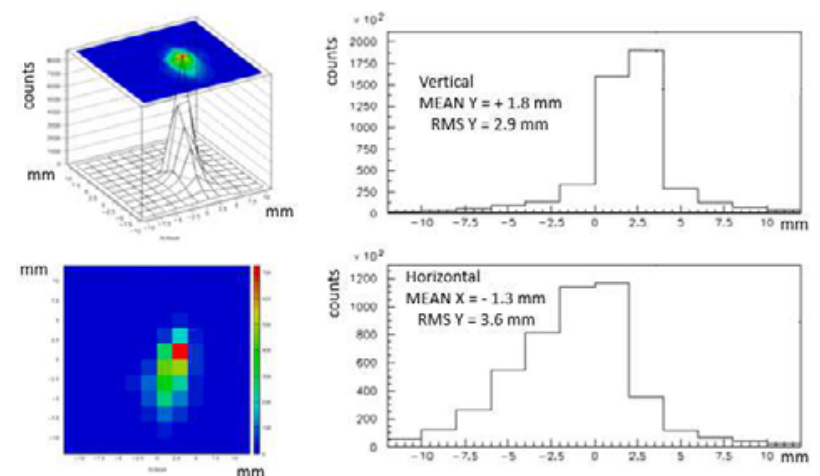

Figure 6. GEM 2D beam imaging showing a non-circular structure.

In addition, some details of the CERF geometry not included in the original simulations might result in small variations in the reference field. For instance, structures like the thin iron roof of the North Experimental Area and the shielding of the beam line adjacent to CERF can cause neutron scattering, which has some impact on the thermal and epithermal parts of the neutron spectrum. The elemental composition of the concrete and iron shielding were also investigated. All of these elements were taken into account in the new simulations currently ongoing with the latest FLUKA version (the development version available at CERN) and will be published in the coming months. In addition to neutrons, all other components of the radiation field (photons, protons, electrons) are being scored.

\section{Conclusions}

Since many years CERF at CERN provides a simulated workplace reference field similar to the ones encountered in the proximity of high-energy accelerators and at cruising altitudes in civil aviation. Its importance is underlined by the ISO standard dealing with simulated workplace neutron fields [1] as well as by an extensive scientific literature reporting on results obtained at CERF. Its wide range of applications extend from the calibration/test of radiation protection instrumentation, to nuclear physics, studies of radiation damage to electronics and more. The accurate characterization of the mixed radiation field by means of Monte Carlo simulations and experimental measurements ensures the reliability of the calibrations and experiments there performed. The undertaken upgrade work has the scope to provide the scientific and industrial community with a further improved and rather unique high-energy reference radiation field.

\section{References}

1. International Standard 12789-1

2. H. Schuhmacher, Radiat. Prot. Dosim. 110, 33 (2004)

3. A. Mitaroff, M. Silari, Radiat. Prot. Dosim. 102, 7 (2002)

4. T. T. Bohlen, F. Cerutti, M. P. W. Chin, A. Fasso, A. Ferrari, P. G. Ortega, A. Mairani, P. R. Sala, G. Smirnov, V. Vlachoudis, Nucl. Data Sheets 120, $211(2014)$

5. A. Ferrari, P.R. Sala, A. Fassò, J. Ranft, CERN2005-10/INFN/TC_05/11, SLAC-R-773, (2005)

6. H.W. Atherton, C. Bovet, N. Doble, G. Von Holtey, L. Piemontese, A. Placci, M. Placidi, D.E. Plane, M. Reinharz, E. Rossa, CERN Yellow Report 80-07 (1980) 
7. A. Ferrari, F.P. La Torre, G.P. Manessi, F. Pozzi, M. Silari, Nucl. Instrum. Meth. A 763, 177 (2014)

8. A. Curioni, R. Froeschl, M. Glaser, E. Iliopoulou, F. P. La Torre, F. Pozzi, F. Ravotti, M. Silari, submitted for publication in Nucl. Instrum. Meth. A

9. C. Birattari, A. Ferrari, C. Nuccetelli, M. Pelliccioni, M. Silari, Nucl. Instrum. Meth A 297, 250 (1990)

10. C. Birattari, A. Esposito, A. Ferrari, M. Pelliccioni, M. Silari, Radiat. Prot. Dosim. 44, 193 (1992)

11. C. Birattari, A. Esposito, A. Ferrari, M. Pelliccioni, M. Silari, Nucl. Instrum. Meth A 324, 232 (1993)

12. C. Birattari, A. Esposito, A. Ferrari, M. Pelliccioni, T. Rancati, M. Silari, Rad. Prot. Dosim. 76, 135 (1998)

13. S. Mayer, D. Forkel-Wirth, M. Fuerstner, H. G. Menzel, M. J. Mueller, D. Perrin, C. Theis, H. Vincke, Radiat. Prot. Dosim. 125, 289 (2007)

14. M. Caresana, M. Helmecke, J. Kubancak, G. P. Manessi, K. Ott, R. Scherpelz, M. Silari, Radiat. Prot. Dosim. 161, 67 (2014)

15. H. Vincke, D. Forkel-Wirth, D. Perrin, C. Theis, Radiat. Prot. Dosim. 116, 380 (2005)

16. E. Aza, M. Magistris, F. Murtas, S. Puddu, M. Silari, J. Instrum. 9, T11005 (2014)

17. K. S. Ytre-Hauge, A. Velure, E. F. Larsen, C. H. Stokkevåg, D. Röhrich, N. Brekke, O. H. Odland, Nucl. Instrum. Meth. A 804, 64 (2015)

18. T. Haninger, P. Kleinau, S. Haninger, Radiat. Prot. Dosim. Doi: 10.1093/rpd/ncw166 (2016)

19. B. Vuković, M. Poje, M. Varga, V. Radolić, I. Miklavčić, D. Faj, D. Stanić, J. Planinić, Appl. Radiat. Isotopes 68, 2398 (2010)

20. I. Jadrníčková, F. Spurný, Radiat. Meas. 43, 683 (2008)

21. M. Kroupa et al., Life Sci. Space Res. 6, 69 (2015)

22. O. Ploc, J. Kubancak, L. Sihver, Y. Uchihori, J. Jakubek, I. Ambrozova, A. Molokanov, L. Pinsky, J. Radiat. Res. 55, 141 (2014)

23. R. K. Piper, J. C. McDonald, M. Silari, F. Pozzi, G. P. Manessi, F. P. La Torre, N. Dinar, to be submitted for publication in Radiat. Prot. Dosim.

24. A. Ferrari, F. P. La Torre, G. P. Manessi, F. Pozzi, M. Silari, Phys. Rev. C 89, 034612 (2014)

25. F. Pozzi, A. Curioni, N. Dinar, F. P. La Torre, G. P. Manessi, M. Silari, submitted for publication in Phys. Rev. C

26. S. Agosteo, F. Pozzi, M. Silari, L. Ulrici, Nucl. Instrum. Meth. B 312, 36 (2013)

27. L. Sarchiapone, M. Brugger, B. Dehning, D. Kramer, M. Stockner, V. Vlachoudis, Nucl. Instrum. Meth. A 581, 511 (2007)

28. R. Garcia Alia, M. Brugger, S. Danzeca, V. FerletCavrois, C. Poivey, K. Roed, F. Saigne, G. Spiezia, S. Uznanski, F. Wrobel, IEEE T. Nucl. Sci. 60, 4142 (2013)

29. A. Cheminet, G. Hubert, V. Lacoste, R. Velazco, D. Boscher, IEEE T. Nucl. Sci. 60, 2411 (2013)

30. www.oecd-nea.org/science/wprs/shielding/ sinbad

31. www.cern.ch/cerf

32. E. Aza, D. Celeste, N. Dinar, E. Iliopoulou, F. P. La Torre, F. Murtas, F. Pozzi, S. Romano, M. Silari, CERN Technical Note, EDMS 1558978, (2015) 